\title{
Light Scattering by Hexagonal Ice Crystals with Distributed Inclusions
}

\author{
R. Lee Panetta*, Jia-Ning Zhang, Lei Bi, Ping Yang, and Guanlin Tang \\ Department of Atmospheric Sciences, Texas A\&M University, College Station, TX 77843, USA \\ ”panetta@tamu.edu
}

\begin{abstract}
Inclusions of air bubbles or soot particles have significant effects on the single-scattering properties of ice crystals, effects that in turn have significant impacts on the radiation budget of an atmosphere containing the crystals. This study investigates some of the single-scattering effects in the case of hexagonal ice crystals, including effects on the backscattering depolarization ratio, a quantity of practical importance in the interpretation of lidar observations. One distinguishing feature of the study is investigation ofscattering properties at a visible wavelength for a crystal with size parameter $(\mathrm{x})$ above 100 , a size regime where one expects some agreement between exact methods and geometrical optics methods. This expectation is generally borne out in a test comparison of how the sensitivity of scattering properties to the distribution of a given volume fraction of included air is represented using (i) an approximate Monte Carlo Ray Tracing (MCRT) method and (ii) a numerically exact pseudo-spectral time-domain (PSTD) method. Another distinguishing feature of the study is a close examination, using the numerically exact InvariantImbedding T-Matrix (II-TM) method, of how some optical properties of importance to satellite remote sensing vary as the volume fraction of inclusions and size of crystal are varied. Although investigation of properties in the $\mathrm{x}>100$ regime face serious computational burdens that force a large number of idealizations and simplifications in the study, an intriguing glimpse is provided of what is evidently a quite complex sensitivity of optical scattering properties to inclusions of air or soot as volume fraction and size parameter are varied.
\end{abstract}

Key words:Scattering, ice crystal, air bubble, soot 


\section{Introduction}

On average, the global ice cloud coverage is approximately $20-25 \%$, whereas the occurrence frequency of these clouds can be as large as $60-70 \%$ in the tropics [1]. Although it is generally recognized that ice clouds play an important role in the terrestrial atmosphere [2-6] and substantial research effort has focused on issues relevant to treatment of clouds in climate models $[7,8]$, the representation of ice clouds in general circulation models (GCMs) is still inadequate [9] in that the cloud distributions simulated by many GCMs are quite different from those inferred from satellite observations [10].Understanding the bulk single-scattering properties of ice clouds, which involve the effect of ice crystal habit (shape) and size distributions,are important for proper interpretation of remote sensing observations and for development of relevant radiation parameterizations in climate models. The recent modeling study [11] demonstrates the importance of the parametrized bulk optical effects of size distributions and habit on simulatedtop-ofatmosphere radiative fluxes. However,even at the level of the optical properties of individual ice crystals, there are weaknesses in our understanding of a number of complicating factors, principal among them being particle shape, surface roughness, and internal inhomogeneity [12-15].

As a follow-up of some previous efforts reported in the literature [16-19], the present study focuses on the single-scattering properties of inhomogeneous ice crystals. Observations have confirmed that some ice crystals contain air bubbles [20]. The voids embedded in ice crystals tend to smooth out the phase function, leading to a featureless scattering pattern. It has been shown ([21],[22]) that the use of an inhomogeneous ice crystal model, namely, ice crystals containing air bubbles, can lead to consistency between simulated polarized reflectance and observations made by the Polarization and Directionality of the Earth's Reflectances (POLDER). It has also been observed that ice crystals in contrails may contain soot particles due to incomplete combustion of fuel by aircraft engines [23]. Furthermore, it has been shown that the atmospheric heating rate associated with a contrail layer is significantly enhanced if soot inclusions are considered [23]. However, with the exception of [19], these studies of the optical properties of ice crystals containing air bubbles and soot particles are essentially based on geometric optics method [16,17] or the geometric-optics surface-wave (GOS) approach [24]. Given the considerable importance of the issues involved, it seems time now to revisit the effects of air and soot inclusions using more rigorous light scattering computational methods.

The pseudo-spectral time-domain (PSTD) and Invariant-Imbedding T-Matrix (II-TM) are "exact" methods, in the sense that they are based on Maxwell's equations rather than phenomenological rationalesor physical approximations made to those equations (note, another exact method is the Discrete Dipole Approximation used in [19].)Results using exact methods are in fact numerical approximations, but, given sufficientcomputer resources, errors in the approximation may in principle be made as small as desired. In reality, however, the state of computer technology at any given time places constraints on what simulations are feasible, and the particular nature of these constraints often suggests use of one exact method over another(e.g.[25] ). We will return to this point in Section 2.By contrast, the Monte Carlo Ray Tracing (MCRT) approach is not an exact method. It, like the method used in [14], is based on geometricoptics approximationsand phenomenological radiative transfer principles rather than on Maxwell's equations. In this case, while there are also numerical errors to be minimized, the convergence that occurs as numerical errors are reduced is to a solution of the approximate model. In general, geometric optics approximations can be justified for large enough size parameters, but begin to break down when the size parameter of interest is smaller than approximately 
100 [26,27].There is no sharply defined boundary of the region in which the MCRT method is reliable, and the only sure test is to compare with a benchmark reference based on a rigorous method. One of the purposes of this study is to compare exact and MCRT results for a choice of ice crystal size in the breakdown region.

We consider ice crystals that are hexagonal prisms, with base having sides of length a and length $\mathrm{L}$ (see Figure 1). We define the crystal's aspect ratio $\rho$ to be

$$
\rho=\frac{L}{2 \alpha}
$$

The increase in severity of computational constraints as particle size increases forces us at several stages in this study to postpone for future work exploration of important sensitivities and keep the focus simply on the effect of inclusions. Our first restriction concerns the value of the aspect ratio. While we expect the optical scattering properties to depend sensitively on the aspect ratio(eg. [27]), we will not attempt to explore that sensitivity here and will in what follows assume unit aspect ratio, $\rho=1$, a special example of what is sometimes referred to as a "compact particle."The same choice was used in the recent study [19] that considered other inclusions beside air and soot, buttook L to be $5 \mu \mathrm{m}$, which is half the smaller of the two sizes we use in all but one case(for Figure 4 below) of PSTD calculations in this study. Other restrictions will be to a single inclusion shape (alwaysspherical), and a single kind of "soot" (also a major simplification see [30], [31]] [32]).

For an incident wave with wavelength $\lambda$ and wavenumber $k=2 \pi / \lambda$, we define the size parameter $x$ of the crystal in terms of D, the diameter of the circumscribing sphere. For a hexagonal crystal D is the longest diagonal of crystal. Our definition is

$x=k \frac{D}{2}=k \frac{L}{2} \sqrt{1+\rho^{-2}}$

We will take the incident radiation to have wavelength $\lambda=0.532 \mu \mathrm{m}$, in the visible range. Thus the larger ice crystal sizes we consider in this study correspond to $x \approx 83.5$ and $x \approx 125$ ( $L=10 \mu \mathrm{m}$ and $\mathrm{L}=15$ $\mu \mathrm{m})$. For this wavelength the index of refraction $n$ for the ice crystal is taken to be $1.3116+1.4824 e^{-9} i[28]$.

With $N$ identical spherical inclusions of radius $r$ (see Figure 1), we define the parameter $V F$ to be the percent of volume occupied by inclusions, in terms of the volume of individual spheres $V_{\text {sph }}$ and the volume of the hexagon $V_{\text {hex }}$, to be

$$
V F=\frac{N V_{s p h}}{V_{\text {hex }}} \times 100 \%
$$

In the following discussions we will refer to $V F$ as the "volume fraction" even though it is literally a volume percentage.

The recent study closest in aims to ours is [19], which used a version of the exact DDA method. Careful comparisons of results using the DDA, PSTD, and II-TM methods have shown the methods to converge with excellent agreement [25], [29]. Beyond numerical methods, and aside from different choices of 
incident wavelengths and consideration of two other inclusions than air and soot, the significant differences between the approach in [19] and ours isthat in [19] a smaller ice crystal size L=5 $\mu \mathrm{m}$, was chosen and a distribution of inclusion sizes was used rather than a single fixed size. We note that for the shorter of two wavelengths $(.65 \mu \mathrm{m})$ considered in [19], that choice ofice crystal size means a size parameter $x$ less than half (41\%) the smaller of two sizes we study in detail: our study is conducted well into the range that was considered too computer-CPU intensive in [19].

\section{Methods}

The essential features of the numerical methods used are briefly sketched here. For details the reader is referred to the papers $[16,33,34]$ for the MCRT, $[25,34]$ for the PSTD, and $[30,36]$ for the II-TM.

\section{1. "Exact" methods}

The PSTD is a time-domain method that gets its name from the use of Fourier transform methods to compute the spatial derivatives in Maxwell's equations, and gains its computational efficiencythrough use of Fast-Fourier-Transform techniques. The time-stepping method used in our PSTD is the commonly used leapfrog method. Figure 2a shows the computational domain with the scattering crystal indicated in green. In the region the scatterer occupies, as well as the "free space" region indicated in white surrounding it, Maxwell's equations hold. In the blue boundary region, the Uni-axial Perfectly Matched Boundary Layer (UPML) modification to Maxwell's equations [33] is applied. The scattered field, indicated in Figure 2a by blue arrows defined as the difference between the total field and an imposed incident field (yellow arrows), is calculated in the computational domain. The scattered near field data are gathered and are typically transformed into far field data by one of two methods: in the "surface integral" method used to obtain the results in this paper, the data are gathered on a so-called "Huygens surface" (indicated by the orange dotted curve in Figure 2a) lying outside the scatterer but within the free space region; in the "volume integral" methodthe input data for the transformation are the field values calculated within the scatterer itself.

A PSTD method can offer definite computational economies over a Finite-Difference Time-Domain (FDTD) method because of the highly efficient and accurate calculation of spatial derivatives. But like the FDTD method, individual calculations must be made for each particular orientation of incident wave with respect to the scatterer. To get what are called "random orientation" results for scattering properties, an average must be taken over a large number of calculations, one for each sampled incident angles, the sampling being fine enough to getnumerical convergence. Since computational time increases rapidly with size parameter, feasibility considerations place limits on size parameters that can be confidently treated. Using current computer technology, for the PSTD method we are using the feasibility limit is in the low hundreds forrandom orientation calculations.

The invariant imbedding T-matrix (II-TM) method is a robust and efficient approach to computing the T-matrix.Unlike the well-known extended boundary conditionT-Matrix method [42],II-TM converts light scattering as a boundary-value problem to an initial-value problem. The method allows directcalculation of random orientation results. Although in principle applicable to arbitrary scatterers, for reasons of computational feasibility the most available T-matrix methods are typically applied to particles with axial symmetries that allow considerable simplification of the calculation. The essential 
idea in the II-TM method is to regard an arbitrarily shaped or configured scatterer as an inhomogeneity imbedded in a surrounding spherical particle with unit index of refraction. The problem of calculating the T-matrix associated with the scatterer is transformed to be a member of a series of related problems that calculate the T-matrices corresponding to a series of partial volumes $V\left(r_{j}\right)$ of the particle; $V\left(r_{j}\right)$ is the volume of the particle within an imaginary spherical domain of the radius $r_{j}(j=1,2,3, \ldots, N)$, $r_{1}=0$, and $r_{N}$ is the radius of the circumscribed sphere (see Figure $2 \mathrm{~b}$ ).Each T-matrix $\mathbf{T}\left(r_{j}\right)$ in conjunction with $V\left(r_{j}\right)$ serves as a stepping-stone in a path to obtaining $\mathbf{T}\left(r_{N}\right)$, which is the T-matrix of the nonspherical particle. Symbolically, this imbedding procedure can be represented as $\mathbf{T}\left(r_{1}\right) \rightarrow \mathbf{T}\left(r_{2}\right) \rightarrow \cdots \rightarrow \mathbf{T}\left(r_{N}\right)$. The numerical algorithm of the procedure was originally obtained from an electric field volume integral equation [28], and further developed in Bi and Yang [27]. The invariant imbedding algorithm to compute the T-matrix is more stable than the extended boundary condition method [42] particularly when the size parameter is large. In the case of a hexagonal particle with an inclusion centered at origin, $\mathbf{T}\left(r_{1}\right)$ can be selected as the T-matrix of the spherical inclusion and computed from the Lorenz-Mie theory to save computational time. Once the T-matrix is known, it is straightforward to compute the corresponding single-scattering properties, namely, the $4 \times 4$ phase matrix, extinction efficiency, and single-scattering albedo.

As mentioned in the introduction, the choice of an exact method is often made on the basis of computational considerations. All the results in this study will be for "random" incident angle, by which is meant an average over incident angles. As just mentioned, in the II-TM method, averages over angles of incidence are performed directly, whereas in the PSTD method calculations must be done for each angle of incidence separately and then the results must be averaged. (The independent angle calculations are typically done in parallel, reducing wall-clock time.) As size parameter increases, demands of computer CPU time and memory increase for each method, but memory requirements increase much more rapidly for the II-TM due to the density of the matrices involved. The overall result is that for smaller size parameters the II-TM demand on computational resources is less than the PSTD demands, and for larger size parameters the PSTD becomes the more economical method. Where the balance tips in favor of the PSTD depends on details of machine configuration, as does the boundary between what is feasible with any numerical method and what is beyond reach. In special cases of particles with symmetries, advantages can be taken in the II-TM that allow its use at larger size parameters, but in general we find the balance on a machine with 12 cores per $24 \mathrm{~GB}$ node begin to shift in favor of the PSTD in the size range 50-60. Accordingly, for numerically exact calculations we use the II-TM for smaller size parameters $(\mathrm{x} \leq 83.5, \mathrm{~L} \leq 10 \mu \mathrm{m})$ and PSTD for larger ones.

As mentioned above, comparisons have already been made between calculations using the II-TM and PSTD methods. In Figure 3 we show phase matrix elements in another such comparison in a format that will be used in subsequent figures. Here the particle is a pristine (no inclusions)hexagonal ice crystal with $\mathrm{x} \approx 20.9(\mathrm{~L}=2.5 \mu \mathrm{m})$. The agreement in results is evidently quite good.

\subsection{MCRT method}

This method is based on a combination of ray-tracing calculations that have modifications to account for Fraunhofer diffraction with a Monte Carlo technique.The Monte Carlo technique is intended to 
reproduce scattering properties of a crystal with small spherical inclusions randomly distributed within the crystal. The mean spacing between inclusions is a parameter $\langle l\rangle$ that is related to the density of identically-sized inclusions: a small value of $<l>$ corresponds to a large number of included particles per unit volume and a large value of $<l>$ to a small number of particles.(The method includes an explicit relation.) Averaging is done over an ensemble consisting of a large number of incident rays, each one treated as follows. Take as an index for ensemble the label $\omega$. Upon incidence at the crystal boundary, the ray refracted inward according to Snel's lawcontinues at most a distance $l_{1}(\omega)$ chosen from a probability distribution with mean $\langle l\rangle$. If in traveling a distance $l_{1}(\omega)$ the ray does not encounter another edge of the face of the crystal, as indicated in Figure 2c, a new direction is chosen from a probability distribution based on the Lorenz-Mie scattering theory, as if the ray had encountered a small sphere, and a new distance $l_{2}(\omega)$ is chosen from the $l$ distribution for propagation of the scattered ray. If $l_{1}(\omega)$ exceeds the distance to another face of the crystal, the ray undergoes another refraction/reflection event at that edge, and a new value $l_{2}(\omega)$ is chosen for the internally reflected ray. Iterations proceed according to these rules until, as usual with ray-tracing calculations, amplitudes of ray signals become negligibly small.

In this approach, there is no single crystal with a definite number of fixed inclusions used, one that could be used as a basis for scattering calculations using one of the exact methods. To compare results obtained with the MCRT method to results with an exact method based on an actual crystal with inclusions, the relation between $\langle l\rangle$ and the number density of inclusions is used. The Lorenz-Mie scattering distribution used to redirect rays in the MCRT calculationassumes a spherical scatterer having the same size parameter as that of the actual spherical inclusions in the exact calculation.

\section{Results}

We first present results from PSTD calculations showing the effect of inclusion of air bubbles on various scattering properties for two different size parameters. We then consider a comparison of PSTD results with MCRT results. We finally look at differences between effects of inclusions of air bubbles and inclusions of soot bubbles. At each stage we are forced to make compromises due to the high computational costs involved in the investigation. In the case of the PSTD calculations, we place a specified number $\mathrm{N}$ of bubbles "at random:" it would be best to do an ensemble of at least 10 experiments, each member having one example of such random placement and average the results over the ensemble. This approach would surely be the best way to compare with the MCRT results. Computational realities would force us to reduce the size parameter to a value like 60, so small as to render the MCRT method highly unreliable. We have chosen instead to do single placement experiments. In the case of the II-TM experiments, where we do a simultaneous consideration of a range of size parameters and volume fractions, we must narrow the focus further to consideration of cases of just one or two bubbles.

\subsection{Volume fraction and bubble size variations: exact (PSTD) calculations}

Figure 4 shows results from PSTD random-orientation calculations for a crystal with $L=10 \mu m(x=83.4)$ in which the number $\mathrm{N}$ of bubbles of radius $0.9187 \mu m(\mathrm{kr} \approx 10.8)$ was either 0 , corresponding to pure ice, 8 , or 16 . The bubbles were placed at random positions, and only one such experiment in each case was done. Since the bubbles were of fixed size, the increase in $\mathrm{N}$ represents an increase in volume fractionfrom 0 through $4 \%$ to $8 \%$. 
The upper left-hand panel shows the effect of increase $\mathrm{N}$ on the phase function $\mathrm{P}_{11}$, with the insert showing effects near forward scattering. Increasing $\mathrm{N}$ clearly results in the elimination of the $22^{\circ}$ and $46^{\circ}$ halos, with the latter halo being the first to disappear as $\mathrm{N}$ increases. It also appears from the figure that the amplitude at exact backscatter is reduced as $\mathrm{N}$ increases. The effect on backscattering amplitude, which cannot easily be seen in the figure but is reported in Table 1, is a decrease when bubbles are added that overall is $46 \%$. From a viewpoint that attributes backscattering to "corner reflections" of rays [39,40], a decrease would be expected if inclusions scatter some of the rays that would otherwise execute corner reflections

Overall, the inclusions reduce the oscillations in the phase function; a similar result was presented in [19]. The effect on asymmetry factor $g$ (see Table 1)isa reduction of about $2 \%$; the values are in an agreement with those of [19] (see their Fig 6) which may be surprising, given differences in size parameter and bubble size distributions, although the agreement in tendency (decrease) of g between what is seen here and in [19] is only true of the initial segment (0-10\%) of the volume fraction range considered in that study. We will examine the effects both on asymmetry factor and backscattering more carefully in section $3 \mathrm{c}$.

The inclusion of bubbles has the effect of decreasing the linear polarization overall. To recognize the effect on depolarization ratio, we recall the relation at exact backscatter $\left(180^{\circ}\right)$

$$
\text { depolarization ratio }=d(r)=\frac{1-r}{1+r}\left(r=\frac{P_{2 n}(180)}{P_{11}(180)}\right) .
$$

Note that

$$
\frac{\partial d}{\partial r}<0,
$$

i.e.a decrease of $\mathrm{P}_{22} / \mathrm{P}_{11}$ at $180^{\circ}$ corresponds to an increase in the depolarization ratio. It can thus be seen from Figure 4, that increasing the volume fraction has the effect of increasing the depolarization ratio: in fact the increase is by $112 \%$ at $\mathrm{VF}=8 \%$ (see Table 1 ).

In spite of the differences in size parameter (a factor of more than 2) that makes detailed comment impossible, and in spite of differences in the manner of producing volume fraction variations, we recognize a number of qualitative similarities between the effects on phase matrix elements shown in Figure 4 and those shown in [19] (their Figure 3, air fraction $=10 \%$ ): for $-\mathrm{P}_{21} / \mathrm{P}_{11}$, increases in a wide range of angles less than about $120^{\circ}$ and decreases at larger angles; for $P_{22} / P_{11}$ and $P_{33} / P_{11}$, reductions most pronounced in a range of angles near side scattering; for $-\mathrm{P}_{34} / \mathrm{P}_{11}$, reductions largest near sidescattering and in a range near back-scattering; and for $\mathrm{P}_{44} / \mathrm{P}_{11}$, reductions in a range of angles extending from side-scattering into the back-scattering region (albeit farther into that region in [19]).

As a simple attempt to determine the effect of "granularity" of inclusions by splitting a given volume fraction over a larger number of bubbles, a calculation was done once using 8 bubbles, and a second time with 16 bubbles, each of the 16bubbleshaving half the volume of bubbles used in the 8bubble case. Figure 5 shows the results. The elimination of the halos appears similar whether the volume fraction is 
distributed over 8 or 16 bubbles, with only a slightly smoother phase function in the $\mathrm{N}=16$ case compared with the $\mathrm{N}=8$ case, suggesting that the eliminationin this case depends little on granularity beyond $\mathrm{N}=8$. Table 1 shows that there isoverall adecrease in the asymmetry factor by about $2 \%$ buta more significant overall increase (53\%) in the backscattering depolarization. While a decrease in backscattering amplitude is clear in going from the pristine case to a volume fraction of $8 \%$, the effect of distributing that $8 \%$ over a number of bubbles is less dramatic.

A second, more cpu-intensive calculation was conducted with a larger crystal having size $L=15 \mu m(x=125)$ to investigate the granularity issue, choosing cases of $\mathrm{N}=1,64$, and 128 while keeping the volume fraction fixed at $8 \%$. At this larger size parameter, where the halos of the host crystal are intrinsically stronger, it becomes clearer that the halo elimination is most effective when the fixed volume fraction is distributed. Effects on other parameters also become clearer. With a larger number of bubbles allowing a more dispersed distribution of the $8 \%$ volume fraction, the reduction in asymmetry parameter is a larger amount of $3.5 \%$. Changes in depolarization ratio are also more dramatic: the increase in the ratio with fixed volume fraction $8 \%$ from $\mathrm{N}=1$ to $\mathrm{N}=128$ is $35 \%$, and the overall change from no inclusions to the volume fraction of $8 \%$ distributed over 128 bubbles is $172 \%$. Again the effect on backscatter amplitude cannot be seen from the figure, it is shown in the table that the amplitude decreases as the $8 \%$ is more widely distributed.

\subsection{Comparison of MCRT with PSTD}

The ray-tracing method underlying the MCRT is expected to perform best for large size parameters, so we compare its performance for the case that $\mathrm{x}=125$. As discussed above, an exact calculation with a given value of N may be compared with a MCRT calculation using the corresponding value of mean distance between inclusions. Results from two such comparisons, using calculations with choices of $\mathrm{N}=0,32$, and 128, are summarized in Figure 7.All bubbles used in the PSTD calculations are spherical and have the same radius, one that corresponds to a volume fraction of $0.0625 \%(=8 \% / 128)$.

The figure shows that overall the MCRT does well in reproducing many features of the phase function, especially the halo regions but in many other regions also. However the method produces results at near backscatter using an interpolation from near-backscatter angles, with results that are hard to interpret. For $\mathrm{N}=0$ and $\mathrm{N}=32$ the phase matrix amplitude increase very rapidly at angles near backscatter: the values at $\theta=179.75^{\circ}$ entered in the table as MCRT backscatter amplitudes, are much larger than the exact PSTD result for $\theta=180^{\circ}$. (The PSTD phase matrix results do not show such dramatic growth near $\theta=180^{\circ}$.) On the other hand, for $\mathrm{N}=128$ the MCRT value at $\theta=179.75^{\circ}$ is a little smaller than the PSTD value. The numerical instability of the MCRT near 180 is known [34] and we are thus hesitant to accept the values given by the MCRT method for backscatter amplitude, or by extension for depolarization ratio.

The agreement in the asymmetry factor, an integral quantity, is better between the two methods. The values are closer, and the qualitative behavior as the volume fraction increases in both cases is the same, a decrease. The overestimate of the asymmetry parameter by the MCRT is consistently in the range $1-2 \%$. 


\subsection{An examination of air and soot inclusions with exact II-TM}

In the previous sections we have shown results for just two crystal sizes, and a few choices of volume fractions. We would like to understand how scattering properties change over ranges of volume fractions and crystal sizes, a computationally daunting task in general, and in particular if the size parameter range exceeds 50 or so. In this section, therefore, we will restrict attention to smaller crystals, ones in the size range of $\mathrm{L}$ between 2 and 10 microns (x between 16.7 and 83.4). In this range it is more computationally economical to use the II-TM method, and it will be possible to consider volume fractions over a range from $1 \%$ to $20 \%$. Even at this smaller crystal size, considerations of computational costs lead us to set aside the issue of how volume fraction is distributed and simply consider a single bubble at the center of the particle. This narrowed focus will make it possible to sample in a range of volume fractions and crystal size rather finely. We will furthermore be able to do this sampling for both air and soot inclusions, taking the index of refraction at wavelength $\lambda=0.532 \mu \mathrm{m}$ to ben $=1.75+0.4453 i$. The contrast between non-absorptive and absorptive inclusions will provide some of our most interesting results.

We begin our comparison of the effects of soot and air inclusions with results for a fixed crystal size at the upper end of the size range $(\mathrm{x}=83.4, \mathrm{~L}=10 \mu \mathrm{m})$ that we sample. We do this at two volume fractions that are chosen in the sampling range. Specifically, Figure 8 compares scattering properties of single spherical inclusions of volume fraction $10 \%$ and $20 \%$ with the same properties in the pristine case. We see immediately from the figure that the halos are progressively smoothed out by either form of inclusion. Looking in more detail we see the interesting result that for angles greater than certain critical one somewhere in the range $65^{0}-70^{\circ}$ (indicated in the figure by the green dotted lines) air and soot inclusions have similar effects of decreasing the phase function amplitude, but forward of that angle and especially in the range of the halos, the inclusions appear have opposite effects. For air inclusions, the smoothing out of halos occurs through an increase in phase function amplitude, while for soot inclusions the smoothing occurs through a decrease. The green arrows have been added to the figures to emphasize the different effects in the forward directions. We have no explanation for this difference in behaviors. We note that the similarity in behaviors in the backscattering direction appears to extend to the exact backscattering amplitude. The exact nature of this similarity in backscattering responses will be examined a little more closely below.

The two types of inclusion have effectson the linear polarization (middle panels in each row of the figure) that are broadly similar to each other. With regard to $\mathrm{P}_{22} / \mathrm{P}_{11}$, shown in the right-most panel in each row, a couple of remarks can be made. In the range of angles $30-120^{\circ}$, there is first of all a behavior that is qualitatively like that seen with the PSTD: an increase of volume fraction decreases the ratio (compare with the upper right-hand panel in Figure 5). Secondly, the behavior in this range of angles seems to be qualitatively opposite to that in the case of soot inclusions (right-most panel in lower row of Figure 8), although the effect in the case of soot appears weaker. Again, we have no explanation for this qualitative difference in behavior as functions of scattering angle.

A closer view of the variation in scattering phase function as the volume fraction of inclusions changes over the range 1-20\% is presented in Figure 9. In that figure, the magenta (pristine case), green and red curves indicate the phase functions plotted in the leftmost panel of Figure 8 as the black, blue, and 
red curves. Note that in the figure, the volume fraction axis is labeled as a true fraction, i.e. without the 100\% multiplier. The same is true in Figures 10 and 11.

Consistent with what was pointed out in the phase function changes in Figure 9, Figure 10 shows that the change with volume fraction occurs in a qualitatively similar manner for air and soot inclusions in the backscattering directions, but the change in the forward scattering directions evidently differs between the cases of air and soot. We note that the case of air shows a rather more intricate pattern of change than does the case of soot.

Figure 10 contrasts, for air and soot inclusions, responses to changes in volume fraction in the range 1$20 \%$ that are seen in efficiency factor, single-scattering albedo, asymmetry factor, and backscattering depolarization ratio. For these results, the crystal size is still fixed at $10 \mu \mathrm{m}(\mathrm{x}=83.4)$; results for air are indicated by blue curves and for soot by magenta curves. The vertical dotted lines in the two right-most panels indicates the volume fraction 8\% that was considered in the upper panels of Figure 8, and was the maximum considered in the PSTD experiments.We note that because the number of inclusions (one) is fixed, a variation of volume fraction means a variation of inclusion size. Thus increases in volume fraction are produced by a different method in these calculations, and this fact must be borne in mind when comparing with PSTD results.

For both air and soot inclusions the efficiency factor shows departures of less than 1\% from the pristine value of 2: in each case the departures appear oscillatory, with more rapid oscillation of extinction efficiency in the case of air in the range of volume fractions considered. This is definitely an interesting aspect of sensitivity to volume fraction, one that the coarser sampling in [19] could not be expected to reveal. We haveno physical explanation for the oscillatory at this time.

For soot inclusions, the sensitivity of single-scattering albedo to volume fraction changes is considerably greater than is the sensitivity of efficiency factor. There is a monotonic decrease of the single-scattering albedo that becomes an almost 25\% reduction when the volume fraction reaches $20 \%$.

Turning to the asymmetry parameter results, the most prominent features are that overall there is a monotonic increase in asymmetry parameter for both air and soot inclusions, and that the sensitivity to volume fraction changes is significantly greater in the case of soot. Looking beyond the overall increase, we see different behaviors shown by soot and air inclusions in the volume fraction range 0$7.5 \%$ (see the circled region in the figure). For soot the initial response is weakbut still increasing, while for air the changes are not monotonic until the volume fraction exceeds about 7.5\%. We do not have an explanation for this complicated response. We note that an increase in the asymmetry parameter in the case of air was also found in [19], once volume fractions increased beyond about 10\%, and that the response of asymmetry parameter to soot inclusion in [19] was qualitatively similar in the range of volume fractions $0-20 \%$, though quantitatively a bit stronger.

For backscattering depolarization ratio, Figure 11 shows that overall there are increases of qualitatively the same magnitude for air and soot inclusions, with a suggestion of slightly different behavior for soot and air inclusions in the range of fractions 5-10\%. We note that the value at volume fraction $8 \%$, approximately .39, is in the range of values (.341-.444 in Table 1) obtained with the PSTD calculations using the same crystal size and volume fraction, but distributing that volume over different numbers of bubbles. 
A comparison in behaviors of soot and air inclusions that brings out the complexity of sensitivity in each case is given is in Figure 11.The results are from calculations that allowed variation of crystal size as well as volume fraction, and they concern changes in three variables of direct relevance to lidar observations: backscattering amplitude, backscattering depolarization ratio, and asymmetry factor, as both $L$ and volume fraction are varied. Note that in the figurethe changes in scattering properties for air and soot inclusions are reported as percent deviations from the values in the pristine crystal case. Note also that the largest size considered, the top value in each panel, is the one we have been discussing in this section.

It is immediately clear is that while overall the responses seen in for air and soot cases are qualitatively similar, looking more closely reveals not only differences in character between the two cases but, as seen in Figure 10, patterns of a complexity for which we cannot account. We comment on only a few of the details here.

Considering first the asymmetry parameter changes, shown in panel (c) of the figure, the qualitative difference we noted above in responses for air and soot cases in the $0-5 \%$ range seems less striking. In each case, for example, the response as crystal size is increased is oscillatory in the range of volume fractions considered. In the range $0-5 \%$ this oscillation may involve an initial increase or an initial decrease in value: the transitions between types of behavior do not always happen at the same crystal size. Evidently at the value $\mathrm{L}=10 \mu \mathrm{m}$, considered in Figure 10, soot behavior was weakly increasing initially while air behavior was clearly decreasing. The oscillatory responses to changes in L are strongest for soot, and are increasingly strong as volume fraction increases.

As volume fraction increases, for the backscattering amplitude the response of changes in size is generally one of monotonic decrease. Significant increases in backscattering are seen but primarily for air inclusions at small crystal size. It is only in this parametric region that the responses of air and soot seem much different. The patterns of response of backscattering depolarization ratio for the cases of soot and air inclusions show similar overall tendencies to increase with volume fractionwith a stronger suggestion of a quasi-oscillatory response to changes in crystal size in the case of air than the case of soot.

\section{Conclusions and caveats}

In this paper we have presented results on spherical inclusions of air and soot in ice crystals. Volume fractions of up to $20 \%$ were investigated for the case of hexagonal crystals of a single aspect ratio. For cases of air and soot inclusions, we considered the effect of variations in total volume fraction, as well as very simple variations in granularity, i.e. the effect of distributing a fixed volume fraction over a varying number of identical spheres.For the larger of two crystal sizes we considered $\mathrm{L}=15 \mu \mathrm{m}(x \approx 125)$, we compared representations of scattering by the PSTD, which solves Maxwell's equations, and the MCRT, which is based on physical optics approximations.

Overall, we found that inclusions suppress the halos that can occur in forward scattering for large enough hexagonal crystals. The suppression occurs increasingly as either volume fraction or 
granularity increases for both cases of air and soot, but in different ways in the two cases. In particular, as volume fraction increases, the halos disappear in the case ofair inclusions by filling in the "valleys" between the peaks in the phase function that correspond to the halos, while the disappearance occurs in the soot case by lowering the peaks. The linear polarization is decreased due to inclusions of either sort. In calculations with variations in volume fraction and granularity, the MCRT gives a good representation of the angular dependence of the phase function, except at exact backscattering angles. The MCRT indicated the same sensitivity of the asymmetry factor to the distribution of a fixed volume of air inclusion over a number of bubbles as that number increases, but seems to predict values $1-2 \%$ larger.

The II-TM calculations showed that in the range of volume fractions 0-20\%, and as L varies over the range 2-10 $\mu \mathrm{m}$ (or size parameter $\mathrm{x}$ over the range 16.7-83.4), the backscattering amplitude increases for $\mathrm{L}<3 \mu \mathrm{m}(\mathrm{x}<25)$ and decreases for $\mathrm{L}>3 \mu \mathrm{m}(\mathrm{x}>25)$. The backscattering depolarization ratio increases for all $\mathrm{x}$, by comparable amounts for soot and air. PSTD calculations conducted using crystal sizes $\mathrm{L}=10 \mu \mathrm{m}, 15 \mu \mathrm{m}(\mathrm{x}=83.4,125)$ also showed that increases in the volume fraction of air inclusions resulted in decreases in the backscattering amplitude. The two sets of calculations also agreed on the tendency of increased volume fraction to increase the depolarization ratio at backscatter. This was in spite of the fact that the increases in volume fraction in the two sets of calculations differed: in the PSTD calculations the volume fraction increase was accomplished by increasing the number of bubbles of fixed size, while in the II-TM calculations using a fixed crystal size the increased volume fraction was accomplished by increasing the size of a single bubble.

The optical property for which the manner of increasing volume fraction clearly did matter was the asymmetry factor. The PSTD calculations, conducted at fixed crystal size, showed decreases of asymmetry factor either as the volume fraction was increased (through adding more bubbles of fixed size) or a given volume fraction was distributed over an increasing number of smaller bubbles. InII-TM calculations conducted at the crystal size $\mathrm{L}=10 \mu \mathrm{m}$, the overall tendency as inclusion volume fraction increased, was to increase the asymmetry factor for both air and soot, the increase in the case of soot being both clearer and more dramatic. However, in the case of air inclusions the behavior of asymmetry factor with increases in volume fraction, in the range of values that was considered in the PSTD calculations, was non-monotonic. Furthermore, in the IITM calculations with fixed volume fraction but varying crystal size (hence correspondingly varying inclusion size), the changes in asymmetry factor showed increasingly strong oscillatory behavior as the value of that fixed volume fraction increased, especially in the case of soot. An understanding of this interestingoscillatory behavior will require further investigation. In any case, it appearsclear that response of the asymmetry factor to inclusions is quite complex and cannot be characterized by a single parameter like volume fraction that contains no information about distribution of that fraction.

The results here, some showing quite striking responses to variations in volume fraction or particle size parameter, must of course be regarded as preliminary, essentially just a glimpse of possible effects of inclusionthat may occur in a much greater variety of circumstances. Only one incident wavelength was considered, only one crystal shape and aspect ratio, only two crystal size parameters, purely spherical inclusions, and one size distribution (uniform) in the case of 
granularity. In the case of random distributions of spherical particles, only a single realization was chosen whereas averaging over an ensemble of realizations might provide a more solid base for conclusions. Exploring more thoroughly any of these parametric variations could easily involve orders of magnitude more cpu time (especially in the case of random inclusion position if the size parameter of the crystal is above 100).

In spite of all these caveats, though, we noted that a number of our findings are consistent with those of the study [19] in a smaller size parameter regime, a study that did include consideration of a range of inclusion sizes and used a different exact numerical method. Specifically, at the slightly different visible wavelength used in [19], in a range of comparable volume fractionssimilar sensitivities to inclusions of air or soot for phase matrix elements. single-scattering albedo, depolarization ratio and asymmetry parameter were reported. This is certainly encouraging, but it is clear that more work involving relaxation of some of the more severe assumptions made in our study will be needed to set its results in proper context.

\section{Acknowledgement}

This study was supported bythe U.S. National Science Foundation under Grant AGS-1338440.We gratefully acknowledge the computational support provided by the Texas A\&M Supercomputing Facility. 


\section{References}

1. Wylie, D. P., and Menzel, W. P., 1999: Eight years of high cloud statistics using HIRS," J. Climate, 12, 170-184.

2. Liou, K. N., 1986: Influence of cirrus clouds on weather and climate process: A global perspective," Mon. Weather Rev., vol. 114, pp. 1167-1199.

3. Stephens, G. L., S. C. Tsay, P. W. Stackhouse, and P. J. Flatau, 1990: The relevance of the microphysical and radiative properties of cirrus clouds to climate and climate feedback," J. Atmos. Sci., vol. 47, pp. 1742-1753.

4. Rossow, W. B., and R. A. Schiffer (1991), ISCCP cloud data products, Bull. Am. Meteorol. Soc., $72,2-20$.

5. Lynch, D. K., K. Sassen, D. OC Starr, and G. Stephens (eds.), 2002: Cirrus, New York: Oxford University Press.

6. Hartmann, D. L., and K. Larson, 2002: An important constraint on tropical cloud-climate feedback. Geophys. Res. Lett., 29.1951, doi:10.1029/2002GL015835.

7. Hahmann, A., D. Ward, and R. Dickinson, 1995: Land surface temperature and radiative fluxes response of the NCAR CCM2/Biosphere-Atmosphere Transfer Scheme to modifications in the optical properties of clouds, J. Geophys. Res., 100(D11), 23239-23252.

8. Del Genio, A. D., M.-S. Yao, W. Kovari, and K.-W. Lo, 1996: A prognostic cloud water parameterization for global climate models. J. Clim., 9, 270-304.

9. Lin, W. Y., and M. H. Zhang, 2004: Evaluation of clouds and their radiative effects simulated by the NCAR Community Atmospheric Model against satellite observations. J. Climate, 17, 3302-3318.

10. Zhang, M. H., et al., 2005: Comparing clouds and their seasonal variations in 10 atmospheric general circulation models with satellite measurements, J. Geophys. Res., 110, D15S02, doi:10.1029/2004JD005021.

11. Baran, A. J., P. Hill, K. Furtado, P. Field, and J. Manners, 2014, A coupled cloud physicsradiation parametrization of the Bulk optical properties of cirrus and its impact on the Met Office Unified Model Global Atmosphere 5.0 configuration, J. Atmos. Sci., 27, 7725-7752.

12. Liou, K. N., Y. Takano, and P. Yang, 2000: Light scattering and radiative transfer by ice crystal clouds: Applications to climate research. Chapter 15 in Light scattering by nonspherical particles: theory, measurements, and geophysical applications, Eds., M. I. Mishchenko, J. W. Hovenier, and L. D. Travis, Academic Press, 417-449.

13. Baran, A. J., 2009: A review of the light scattering properties of cirrus. J. Quant. Spectrosc. Radiat. Transfer, 110, 1239-1260.

14. Baran, A. J., 2012: From the single-scattering properties of ice crystals to climate prediction: A way forward. Atmos. Res., 112, 45-69.

15. Smith, H. R., P. J. Connolly, A. J. Baran, E. Hesse, A. R. D. Smedley, and A. R. Webb, 2015, Cloud chamber laboratory investigaions into scattering properties of hollow ice particles, J. Quant. Spectorsc. Radiat. Transfer, 157, 106-118.

16. Macke, A., M. I. Mishchenko, and B. Cairns ,1996, The influence of inclusions on light scattering by large ice particles, J. Geophys. Res., 101, 23311-23316. 
17. Xie, Y., P. Yang, G. W. Kattawar, P. Minnis, Y. Hu, 2009: Effect of the inhomogeneity of ice crystals on retrieving ice cloud optical thickness and effective particle size, J. Geophys. Res. 114, D11203, doi:10.1029/2008JD011216.

18. Liou, K. N., Y. Takano, Q. Yue, and P. Yang, 2013: On the radiative forcing of contrail cirrus contaminated by black carbon, Geophys. Res. Lett., 40, 1-7, doi:10.1002/grl.50110.

19. Hong, G. and P. Minnis, 2015, Effects of spherical inclusions on scattering properties of small ice cloud particles, J. Geophy. Res., 120,2951-2989, doi:10.1002/2014JD022494.

20. Tape, W. (1994), Atmospheric halos, American Geophysical Union, Washington, D. C., USA.

21. C.-Labonnote, L., G. Brogniez, J. C. Buriez, and M. Doutriaux-Boucher (2001), Polarized light scattering by inhomogeneous hexagonal monocrystals: validation with ADEOS-POLDER measurements, J. Geophy. Res., 106, 12139-12155.

22. Baran, A. J. and L. C. Labonnote ,2006, On the reflection and polarization properties of ice cloud, J. Quant. Spectrosc. Radiat. Transfer, 100, 41-54.

23. Petzold, A., and A. Döpelheuer ,1998, Reexamination of black carbon mass emission indices of a jet engine, Aerosol Sci. Technol., 29, 355-356.

24. Liou, K. N., Y. Takano, and P. Yang,2010, On geometric optics and surface waves for light scattering by spheres. J. Quant. Spectrosc. Radiat. Transfer, 111, 1980-1989.

25. Liu, C., L. Bi, R. L. Panetta, P. Yang, and M. A. Yurkin, 2012: Comparison between the pseudospectral time domain method and the discrete dipole approximation for light scattering simulations. Opt. Express, 20, 16763-16776.

26. Mishchenko, M. I., and A. Macke, 1999: How big should hexagonal ice crystals be to produce halos?, Appl. Opt. 38, 1626-1629.

27. Yang, P., and Q. Fu, 2009: Dependence of ice crystal optical properties on particle aspect ratio, J. Quant. Spectrosc. Radiat. Transfer, 110, 1604-1614.

28. Warren SG, Brandt RE. ,2008, Optical constants of ice from the ultraviolet to the microwave: A revised compilation. J. Geophys. Res, 113, D14220.

29. Bi, L. and P. Yang, 2014, Accurate simulation of the optical properties of atmospheric ice crystals with the invariant imbedding T-matrix method, J. Quant. Spectrosc. Radiat. Transfer, 138, 17-35.

30. Andreae, M. O. and A. Gelenser, 2006, Black carbon or brown carbon? The nature of lightabsorbing carbonaceous aerosols, Atmos. Chem. Phys, 6, 3131-3148.

31. Bond, T. C., G. Habib, R. Bergstrom, 2006,Limitations in the enhancement of visible light absorption due to mixing state, J. Geophys. Res., 111, D20211.

32. Cappa, C. D. , T. B. Onasch, P. Massoli, D. R. Worshop, T. S. Bate, E. S. Cross, P. Davidovits, J. Hakata, K. L. Hayden, B. T. Jobson, K. R. Kolesar, D. A. Lack, B. M. Lerner, S-M. Li, D. Mellon, I. Nuaaman, J. S. Olfert, T. Petaja, P. K. Quinn, C. Song, R. Subramanian, E. J. Williams, and R. A. Zaveri, 2012, Radiative Absorption Enhancements Due to the mixing State of atmospheric black carbon, Science, 337, 1078-1081.

33. Wendling, P., Wendling and H. K. Weickmann, 1979, Scattering of solar radiation by hexagonal ice crystals. Appl. Optics., 18: 2663-71.

34. Macke, A. J. Mueller, and E. Raschke (1996), Single scattering properties of atmospheric ice crystal, J. Atmos. Sci., 53, 2813-2825.

35. Liu, Q. H., 1997: The PSTD algorithm: A time-domain method requiring only two cells per wavelength, Microw. Opt. Techn. Let., 15, 158-165. 
36. Johnson B.R., 1988, Invariant imbedding T-matrix approach to electromagnetic scattering. Appl Opt , 27:4861-73.

37. Panetta, R. L., C. Liu, P. Yang, 2013, A pseudo-spectral time domain method for light scattering computation, in Light Scattering Reviews 8 (ed. A. Kokhanovsky), Springer.

38. Gedney, S. D., 1996, An anisotropic perfectly matched layer-absorbing medium for the truncation of FDTD lattices, IEEE Trans. Antennas Propag. 44 (12), 1630-1639.

39. Borovoi, A. G., V. Kustova, U. G. Oppei, 2005, Light backscattering by hexagonal ice crystal particles in the geometric optics approximation, Opt. Eng. 44(7), 071208.

40. Borovoi, A. G., A. Konoshonkin, N. Kustova, H. Okamoto, 2012, Backscattering Mueller matrix for quasi-horizontally oriented icd plates of cirrus clouds: application to CALIPSO signals, Opt. Express, 20(27), 28222-28233.

41. C.-Labonnote, L., G. Brogniez, M. Doutriaux-Boucher, J.-C. Buriez, J.-F. Gayet, H. Chepfer, 2000, Modeling of light scattering in cirrus clouds with inhomogeneous hexagonal monocrystals. Comparison with in-situ and ADEOS-POLDER measurements, GRL, 27 (1), 113-116.

42. Waterman PC. , 1965, Matrix formulation of electromagnetic scattering. Proc IEEE, 53:80512. 


\begin{tabular}{|c|c|c|c|c|c|c|}
\hline Figure & $\mathrm{x} \quad$ (L) & $\mathrm{N}$ bubbles & Vol Frac & Asymmetry & Depolarization & Backscatter \\
\hline \multirow[t]{3}{*}{4} & $83.4(10)$ & 0 & 0 & .769 & .209 & .676 \\
\hline & & 8 & 4 & .762 & .289 & .423 \\
\hline & & 16 & 8 & .754 & .444 & .362 \\
\hline \multirow[t]{4}{*}{ 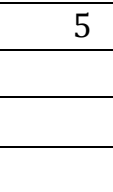 } & $83.4(10)$ & 2 & 8 & .767 & .341 & .401 \\
\hline & & 4 & 8 & .768 & .497 & .380 \\
\hline & & 8 & 8 & .757 & .417 & .362 \\
\hline & & 16 & 8 & .754 & .444 & .362 \\
\hline \multirow[t]{4}{*}{6} & 125 & 0 & 0 & .761 & .200 & .462 \\
\hline & & 1 & 8 & .742 & .404 & .499 \\
\hline & & 64 & 8 & .719 & .504 & .321 \\
\hline & & 128 & 8 & .707 & .545 & .315 \\
\hline \multirow[t]{3}{*}{7} & $125(15)$ & 0 & 0 & $.761, .774$ & $.200, .506$ & $.462,6.99$ \\
\hline & & 32 & 2 & $.740, .753$ & $.378, .472$ & $.447,1.29$ \\
\hline & & 128 & 8 & $.707, .715$ & $.546,1.12$ & $.315, .268$ \\
\hline
\end{tabular}

Table 1. Integral scattering properties for Figures 4-7. Figure 7 shows comparisons of results obtained from PSTD and MCRT, and where pairs of data appear in columns 5-8, the first value is from the PSTD calculation and the second from the MCRT calculation. 


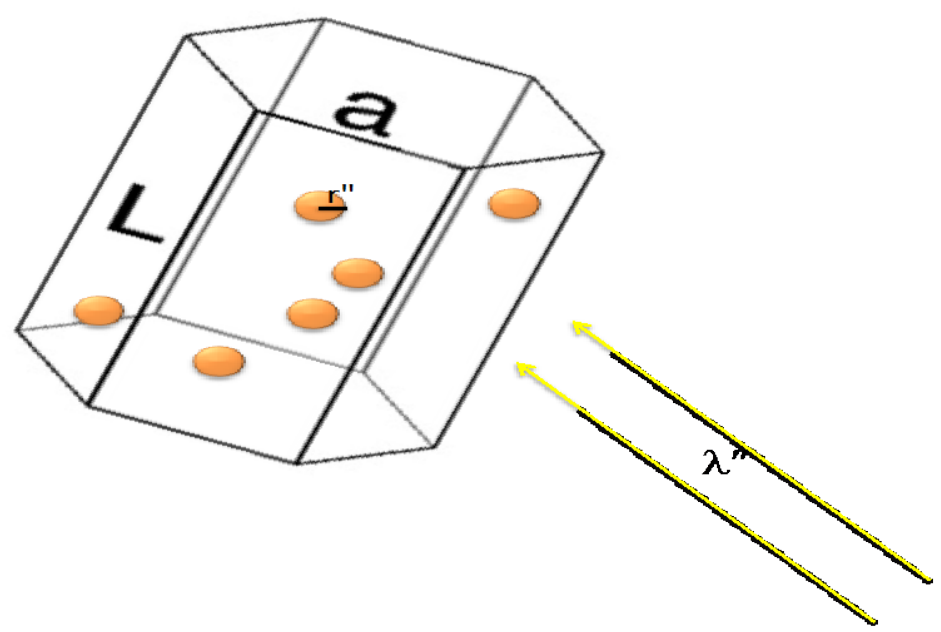

Figure 1. Hexagonal crystal of length $L$ and basal edge length a having spherical inclusions of radius $r$. 


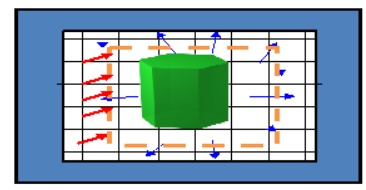

(a)

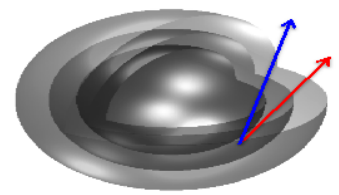

(b)

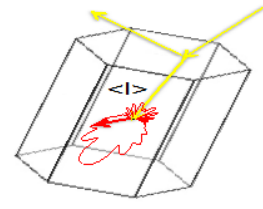

(c)

Figure 2. (a) The computational domain for the PSTD; (b) the shells involved in the II-TM; and (c) the geometry involved in the MCRT. See text for details 

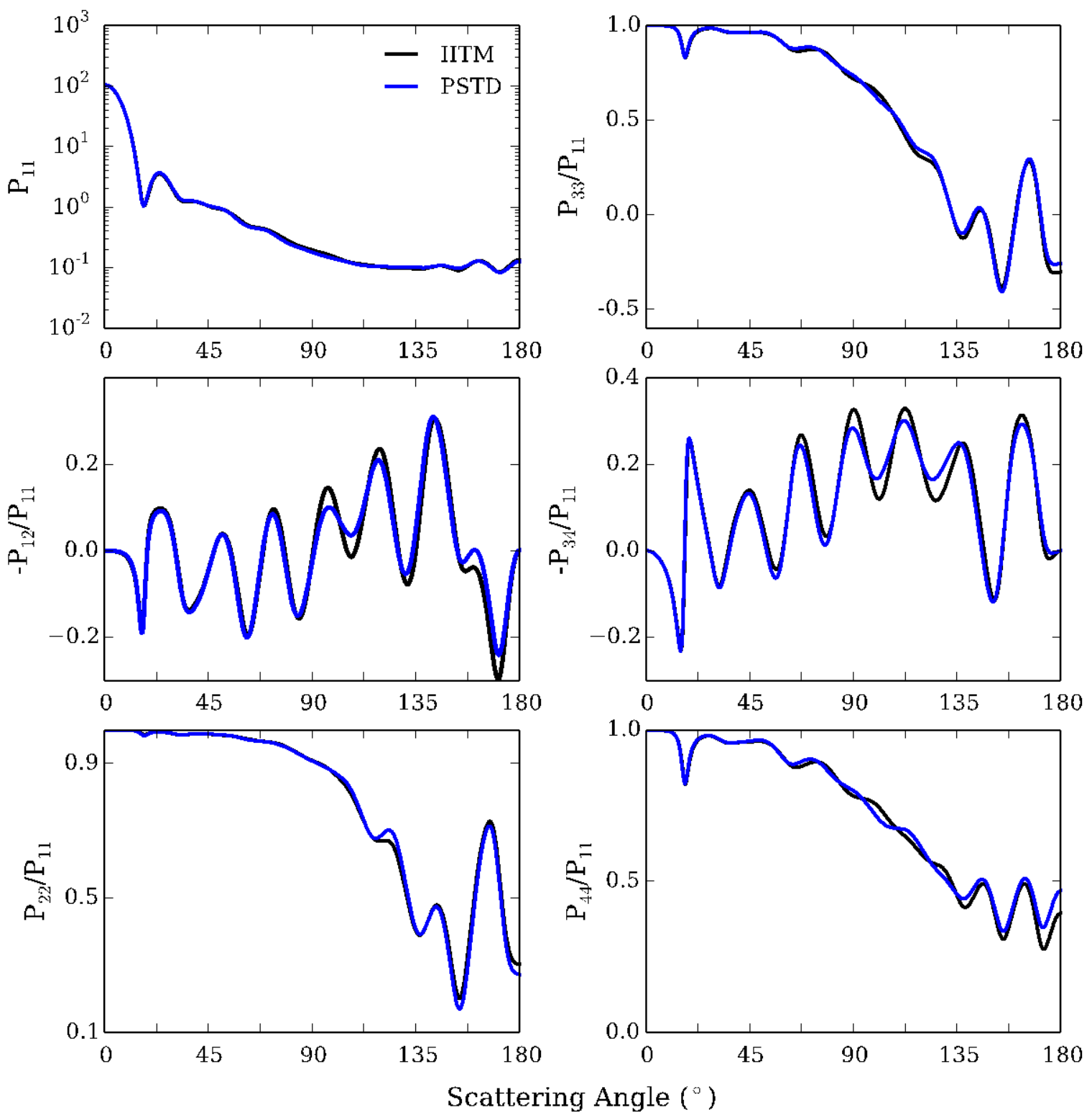

Figure 3. Comparison of scattering properties of pristine hexagon as computed using the PSTD and II-TM methods. The particle size parameter $\mathrm{x}=20.9(\mathrm{~L}=2.5 \mu \mathrm{m})$. 

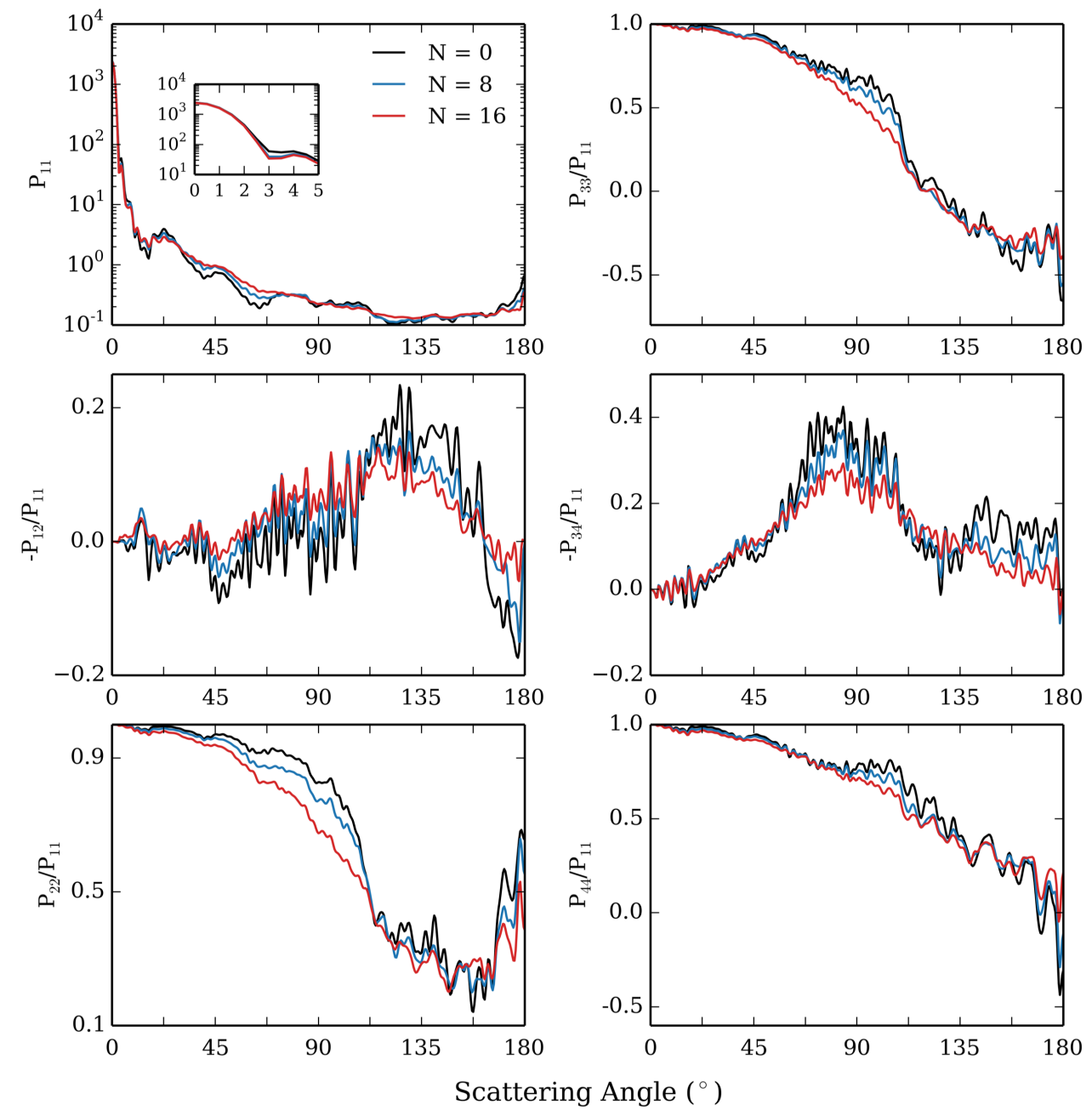

Figure 4. Effects of increasing volume fraction by increasing number of bubbles of fixed size $(\mathrm{r}=0.9187 \mu \mathrm{m})$ : largest volume fraction is $8 \%$. Insert in $\mathrm{P}_{11}$ panel shows detail near direct forward scattering angles. Crystal size parameter $\mathrm{x}=83.4(\mathrm{~L}=10 \mu \mathrm{m})$. 

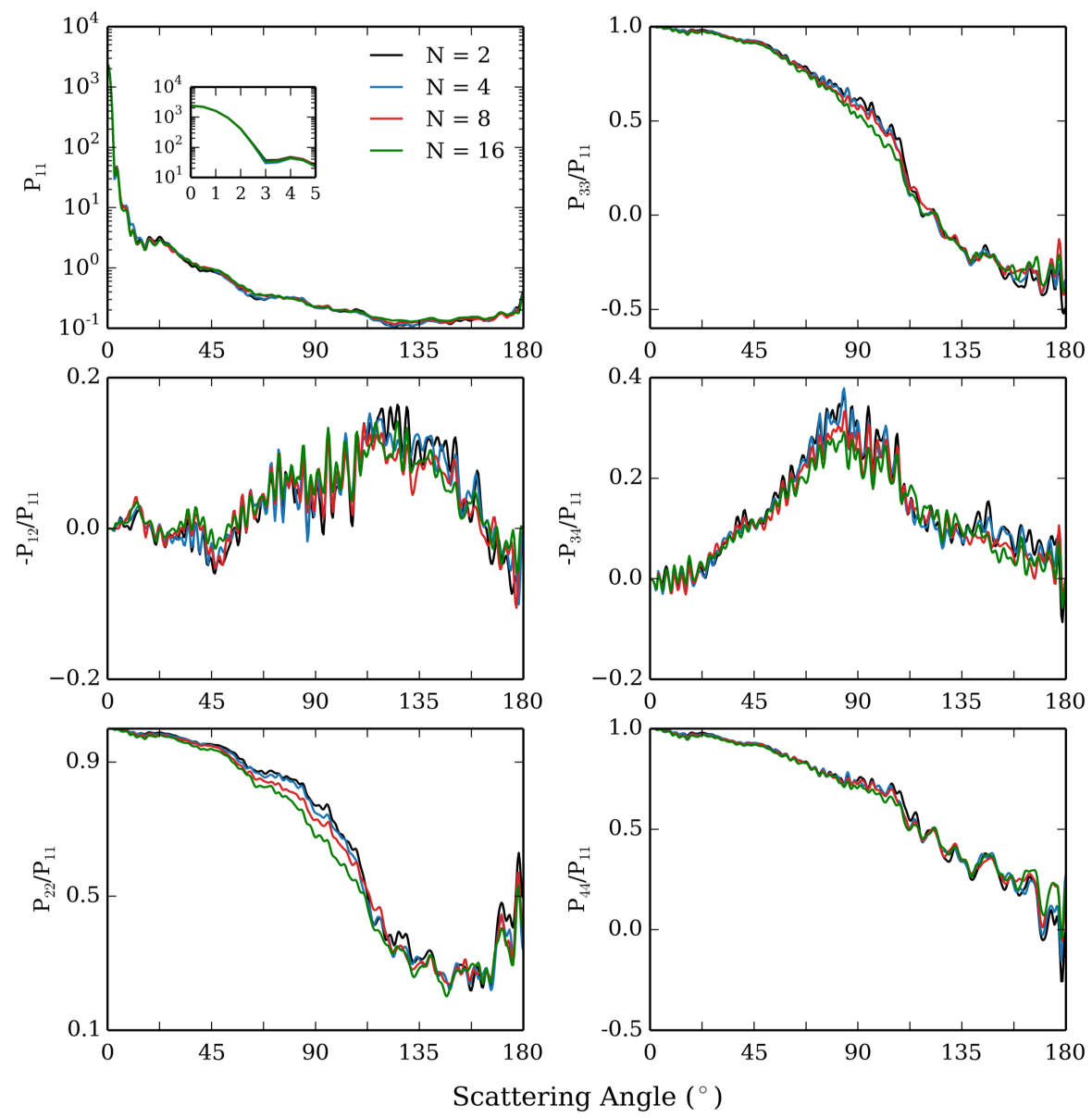

Figure 5. Effects of distributing fixed volume fraction of $8 \%$ over increasing number $\mathrm{N}$ of equalsized bubbles.Crystal size parameter $\mathrm{x}=83.4(\mathrm{~L}=10 \mu \mathrm{m})$. 

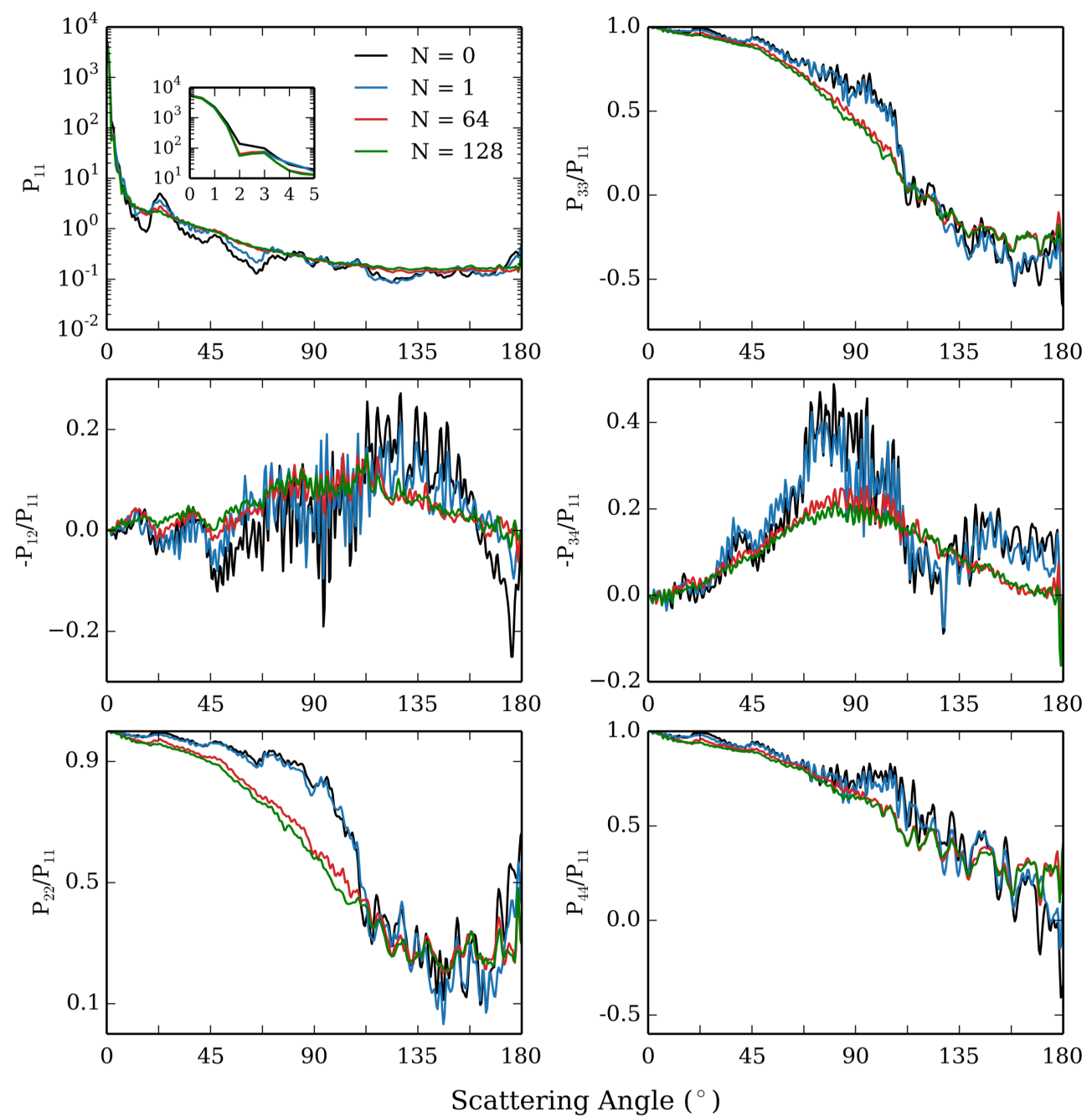
Figure 6. Fixed volume fraction (8\%), number of air bubbles $\mathrm{N}=0,1,64,128$. Crystal size parameter $\mathrm{x}=125(\mathrm{~L}=15 \mu \mathrm{m})$. 

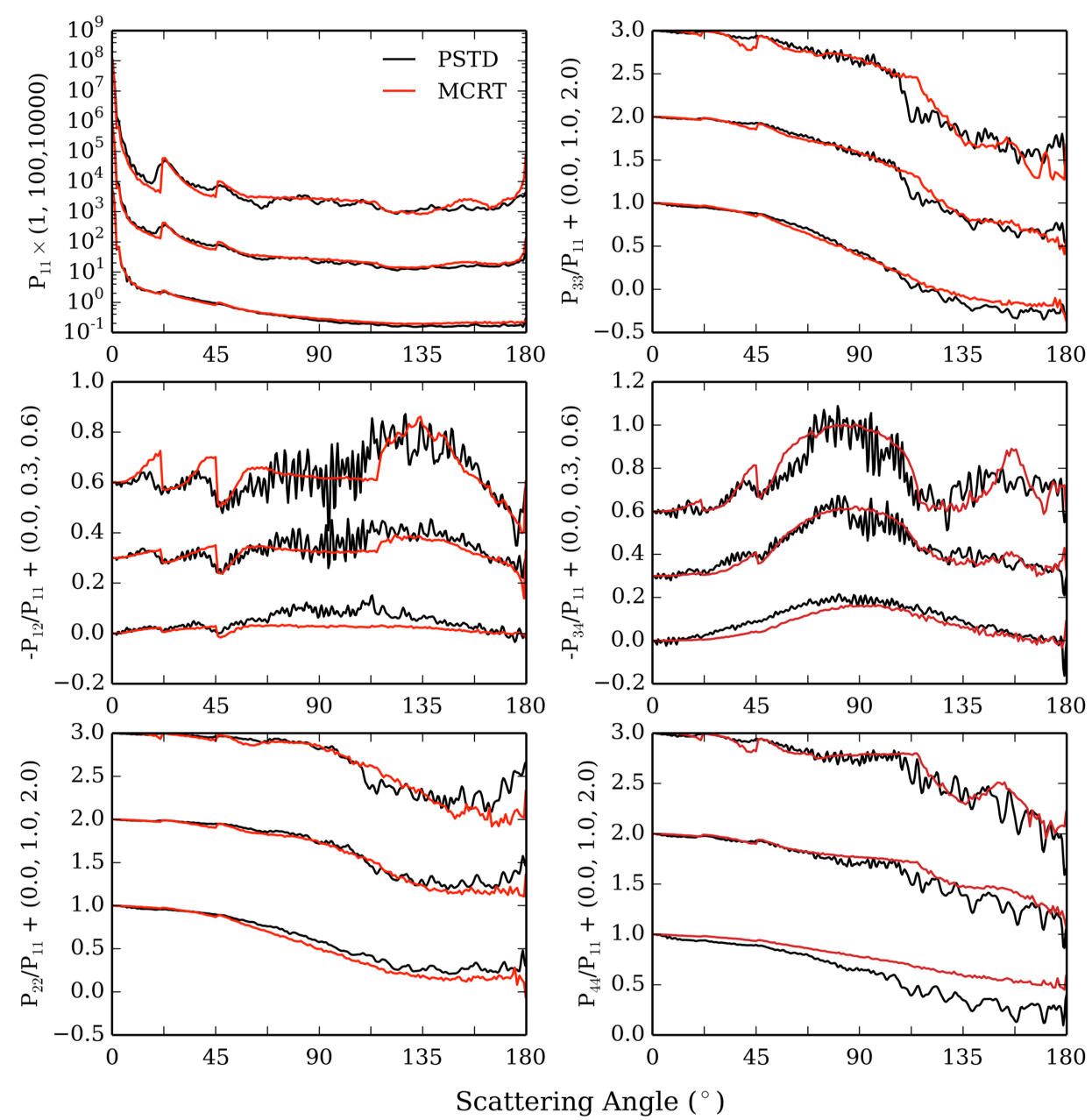

Figure 7. Comparison of MCRT and PSTD results for air bubble inclusions. The equal-sized bubbles have radii corresponding to a volume fraction of $0.0625 \%$; choices of $\mathrm{N}=(0,32,128)$ correspond to $\mathrm{VF}=(0,2 \%, 8 \%)$. Crystal size parameter is $\mathrm{x}=125(\mathrm{~L}=15 \mu \mathrm{m})$. 
(a)
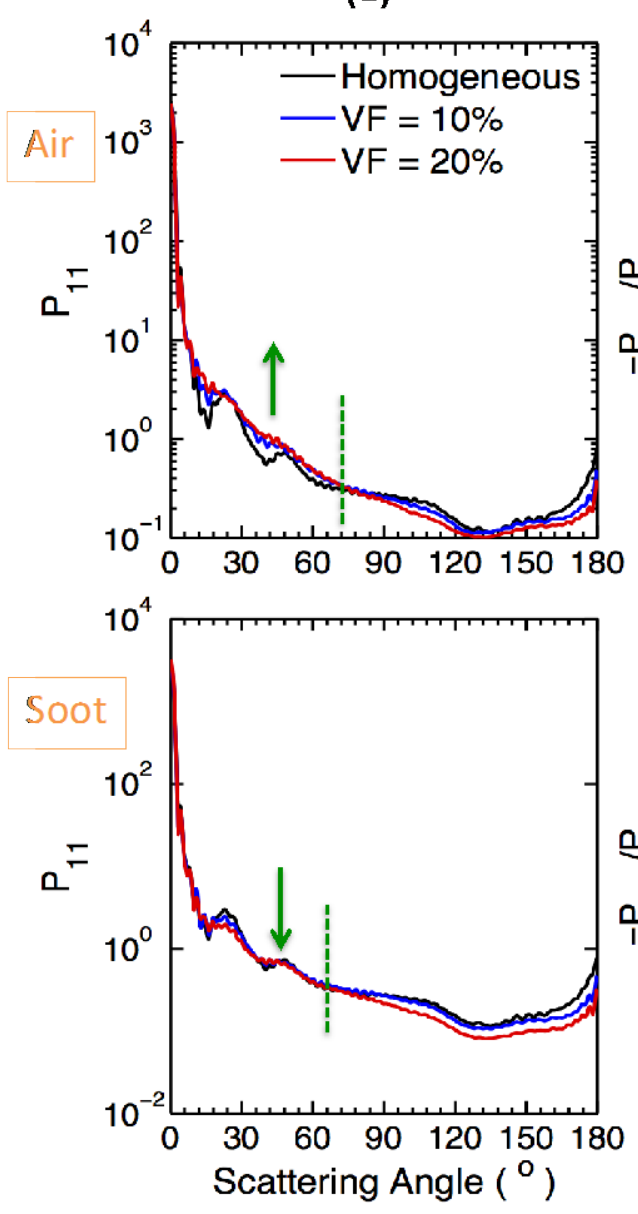

(b)
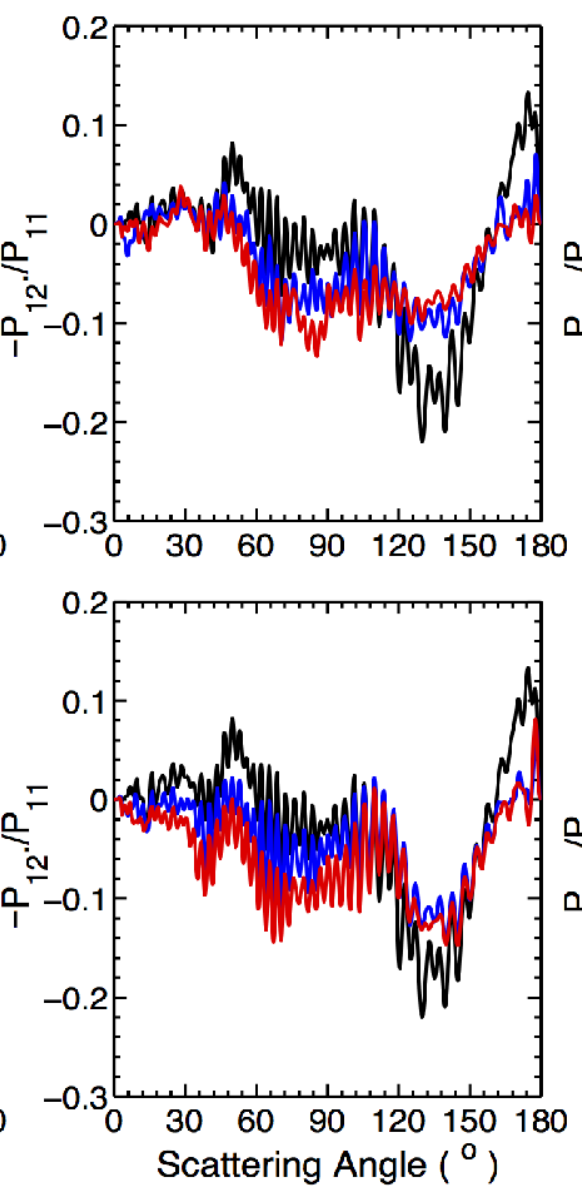

(c)
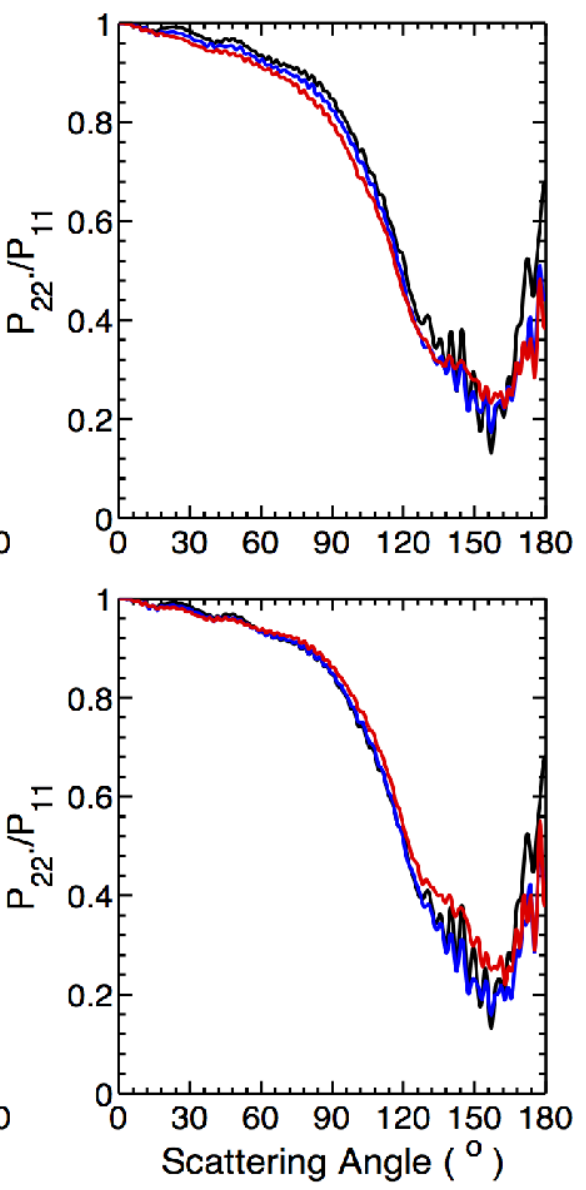

Figure 8. (a) Phase function, (b) -P12/P11, and (c) P22/P11 for different volume fractions of air and soot inclusions. Crystal size parameter is $\mathrm{x}=83.4(\mathrm{~L}=10 \mu \mathrm{m})$. Meanings of green dashed lines and arrows are explained in text. 

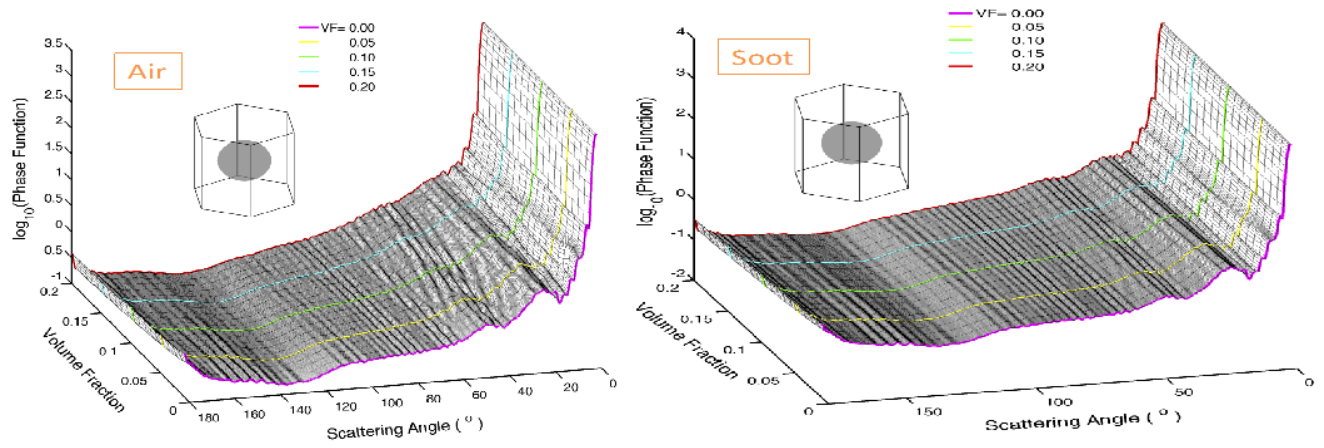

Figure 9. Variation of phase function as the volume fraction of a single spherical inclusion of either air or soot increases from 0 to $10 \%$. Notice the change perspective from that of other figures: the scattering angle decreases to the right. The volume fraction axes are scaled as $\mathrm{VF} / 100 \%$, i.e. as actual fractions of volume. 

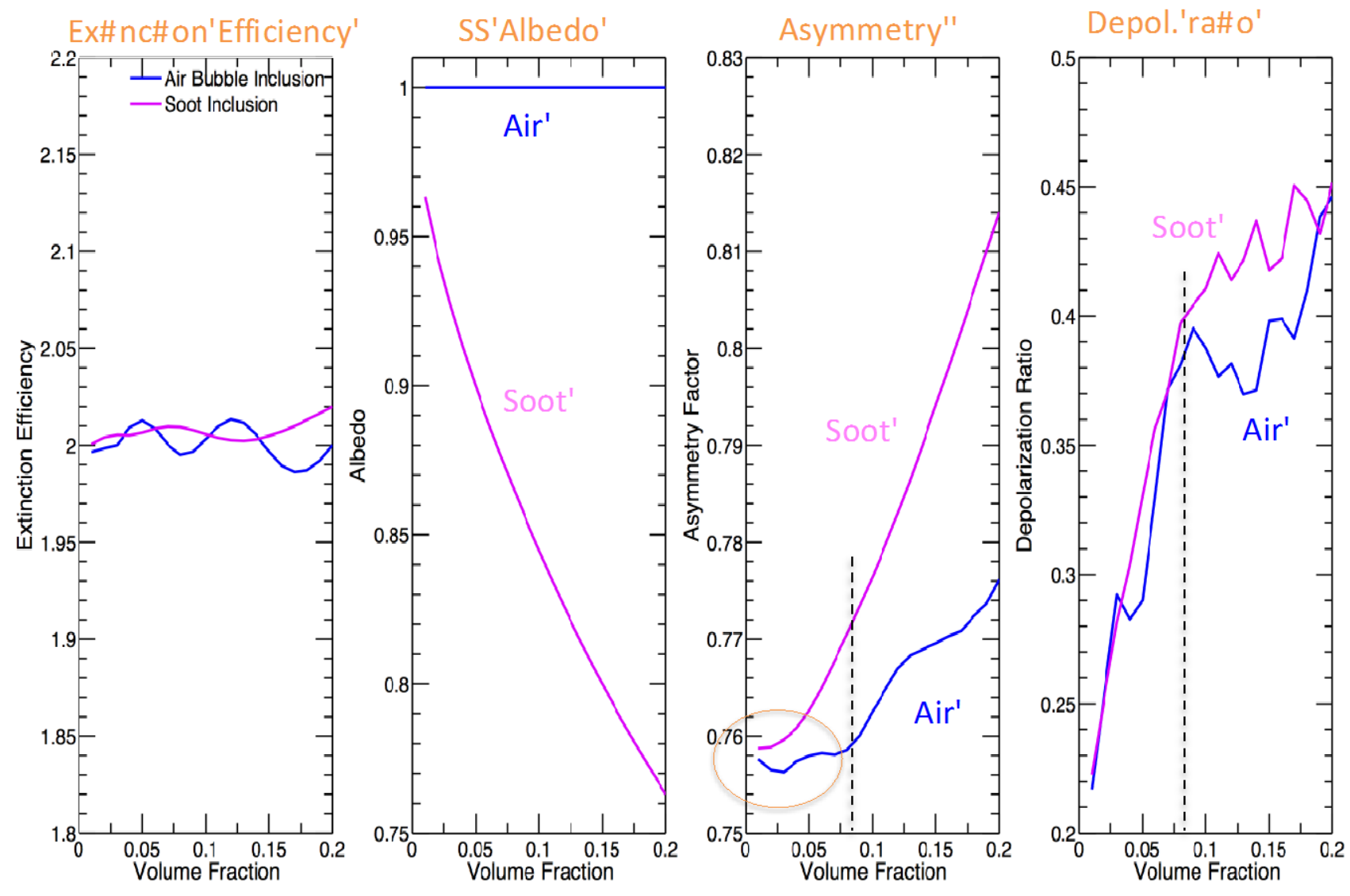

Figure 10. For single spherical inclusions of air and soot, effects of increasing volume fraction on extinction efficiency, single scattering albedo, asymmetry factor, and backscattering depolarization ratio.Crystal size parameter is $\mathrm{x}=83.4(\mathrm{~L}=10 \mu \mathrm{m}$.) The volume fraction axes are scaled as in Figure 9. 
(a)
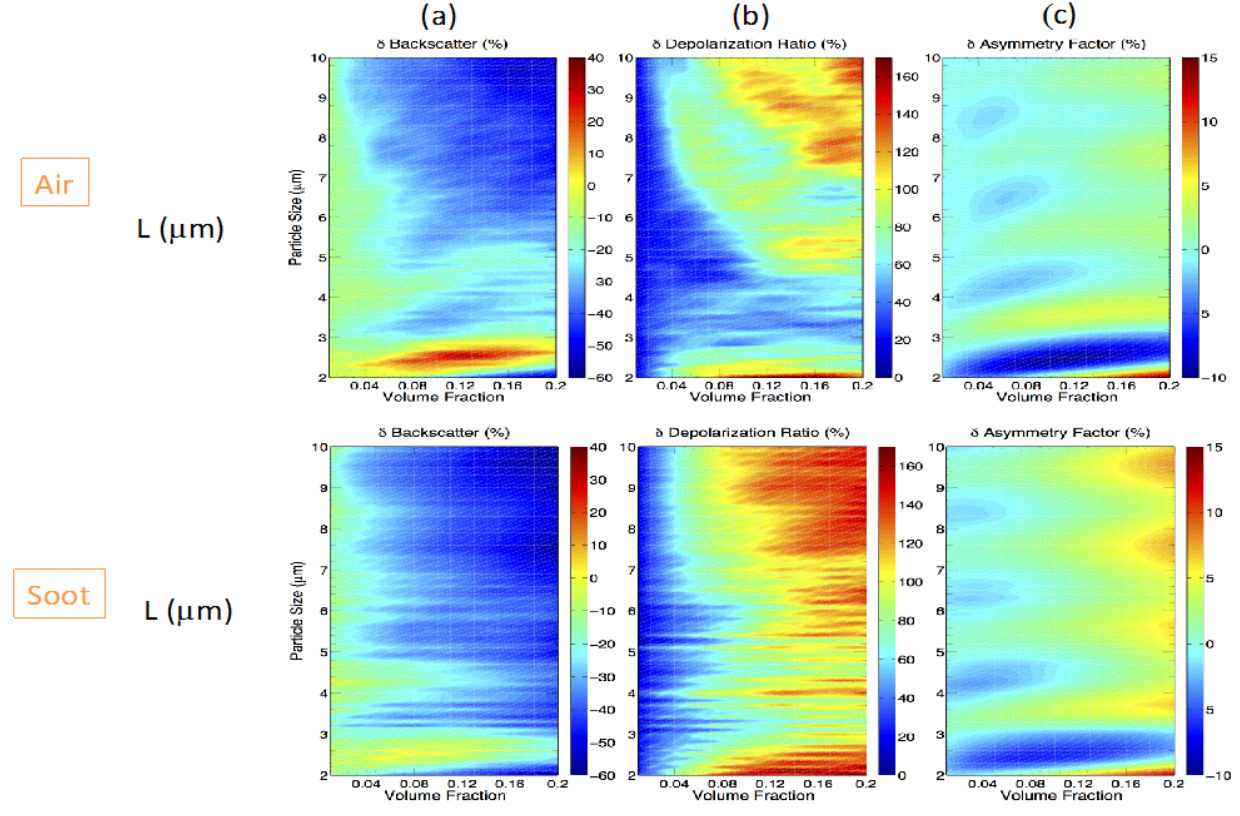

Figure 11. Changes, expressed as a percentage to (a) backscattering amplitude, (b) backscattering depolarization ratio, and (c) asymmetry factor, due to changes in volume fraction (horizontal axis in each plot) of a single spherical inclusion. The vertical axis in each plot is labeled with the physical size L in microns of the crystal. The volume fraction axes are scaled as in Figure 9. 